\title{
MORBIMORTALIDADE POR TRAUMATISMO CRÂNIO-ENCEFÁLICO NO MUNICÍPIO DE SÃO PAULO, 1997
}

\author{
MARIA SUMIE KOIZUMI*, MARIA LÚCIA LEBRÃO**, \\ MARIA HELENA PRADO DE MELLO-JORGE** VANESSA PRIMERANO***
}

\begin{abstract}
RESUMO - Estudos epidemiológicos sobre traumatismos crânio-encefálicos (TCE) são poucos,e mais escassos ainda os que permitem análises comparativas da magnitude desta problemática, devido obstáculos metodológicos. No geral, os estudos são de trauma cefálico (incluem envoltórios e conteúdo craniano) mas reportam-se como de TCE, dada a dificuldade de mútua exclusão. É usual também estarem centrados em níveis específicos de gravidade, somente em vítimas hospitalizadas, ou de acordo com a causa externa como o acidente de trânsito. Respeitadas tais limitações, este estudo teve como objetivo estimar a incidência de TCE nas vítimas residentes e internadas na rede hospitalar do Município de São Paulo, em 1997, bem como, estimar a mortalidade por TCE dentre as mortes por causas externas, neste mesmo período e local. O material de estudo constou de dados do Ministério da Saúde relativos às saídas hospitalares analisadas a partir das Autorizações de Internação Hospitalar (AIH) e dados de óbitos por causas externas contidas no banco de dados do Programa de Aprimoramento das Informações de Mortalidade (PROAIM). Constatou-se que 29717 pacientes foram internados devido lesões e envenamentos e destes, $3.635(12 \%)$ tinham TCE; a taxa de internação foi 0,36/1 000 habitantes e a de mortalidade hospitalar foi $10 \%$. O coeficiente de mortalidade por causas externas foi de 87,3/100 000 habitantes. Estima-se que a taxa de mortalidade por TCE seja no mínimo de 26,2 e no máximo de 39,3/100 000 habitantes.
\end{abstract}

PALAVRAS-CHAVES: epidemiologia, morbidade, mortalidade, traumatismo crânio-encefálico.

\section{Morbidity and mortality due to traumatic brain injury in São Paulo city, Brazil,1997}

ABSTRACT - There are few epidemiological studies on traumatic brain injury (TBI), and those that allow broad comparative analyses of this problem are even more scarce, due to methodological obstacles. Usually, the studies address head injury (they include the cranial envelopments and content) but are reported as TBI, given the difficulty of mutual exclusion. It is also common for them to be centered at specific severity levels, only for hospitalized victims or according to the external cause, such as traffic accidents. With full respect to these limits, this study had as its objective the estimation of the TBI incidence in patients resident and in-hospital, in the hospital network in the city of São Paulo, Brazil, in 1997, as well as the estimation of TBI-caused mortality amongst external causes, at this same time and locale. Data for the study consisted of Ministério da Saúde data on hospital discharges, analyzed based on Autorizações de Internação Hospitalar (AIH) and obituary data on external causes, contained in the Programa de Aprimoramento das Informações de Mortalidade (PROAIM) data base. It was found that 29717 patients were hospitalized due to lesions and poisoning; of these $3635(12 \%)$ had TBI; the hospitalization rate was $0.36 / 1000$ pop.; and hospital mortality was $10 \%$. The mortality coefficient due to external causes was $87.3 / 100000$ pop. Minimum TBI mortality rate is estimated at a minimum of 26.2 and a maximum of $39.3 / 100000$ pop.

KEY WORDS: epidemiology, morbidity, mortality, head injury.

A obtenção de dados tendo como referência a natureza da lesão, no caso o traumatismo crânio-encefálico (TCE), é sempre difícil, mesmo nos países desenvolvidos, devido a problemas de

*Professor Titular da Escola de Enfermagem (EE) da Universidade de São Paulo (USP). **Professor Associado da Faculdade de Saúde Pública da USP - Departamento de Epidemiologia; ***Bolsista IC- CNPq, Aluno de graduação em Enfermagem. Aceite: 11-outubro-1999.

Dra. Maria Sumie Koizumi - GENT, Grupo de Estudos em Neurotrauma, EEUSP - Av. Dr. Enéas de Carvalho Aguiar 419 - 05403-000 São Paulo, SP - Brasil. 
Quadro 1. Alguns estudos selecionados sobre incidência do TCE, nos Estados Unidos da América.

\begin{tabular}{|c|c|c|c|}
\hline Pesquisa & Abrangência & Ano & Inclusões \\
\hline Olmsted County & Hospital & $1935-1974$ & Não informado \\
\hline $\begin{array}{l}\text { National Head and } \\
\text { Spinal Cord Injury Survey }\end{array}$ & EUA & 1974 & $\begin{array}{c}\text { CID-8: } 253.9,293.5,309.2,310.0,311.1 \\
315.1,345,347.9,800,803,804, \\
850-854,904.1,950,952,995.1,995.2\end{array}$ \\
\hline San Diego County & várias fontes & 1978 & CID-8: $800,801,805,806,850-854$ \\
\hline Bronx, NY & hospital ( 24 horas) & $1980 / 1981$ & CID-9CM: 800, 801, 803-806, 850-854 \\
\hline North Central Virginia & 12 cidades & 1978 & Não informado \\
\hline San Diego County & Hospital & 1981 & $\begin{array}{c}\text { CID-9: } 800-804,344,345,850-854 \\
\quad 873,905,907,910,920\end{array}$ \\
\hline Chicago Area & Hospital & $1979 / 1980$ & $\begin{array}{l}\text { CID-9CM: } 800-804,830 \text {, } \\
850-854,873,920,959.0\end{array}$ \\
\hline Rhode Island & Hospital & $1979 / 1980$ & CID-9CM: 800.0 \\
\hline Maryland & 56 hospitais & 1986 & CID-9CM: $800,801,803,804,850-854$ \\
\hline United States & $\begin{array}{l}\text { National Health } \\
\text { Interview Survey }\end{array}$ & 1977-1981 & $\begin{array}{l}\text { CID-9CM: 800.0-801.9, } \\
803.0-803.9,850.0-854.9\end{array}$ \\
\hline
\end{tabular}

Fonte: Kraus $\mathrm{JF}^{3}$.

ordem metodológica. Os dados epidemiológicos dos TCE são incompletos, pois não há informações que abranjam todos os pacientes traumatizados, tanto nos aspectos de morbidade como de mortalidade. Conforme regras internacionais, nos casos de acidentes e violências, o sistema para coleta de informações utiliza referenciais diferentes para morbidade e mortalidade. Para a morbidade, o referencial é a natureza da lesão, ou seja, fraturas, luxações, ferimentos, contusões e envenenamentos, como exemplos. Para a mortalidade, o referencial é a causa externa, o acidente ou a violência que originou o evento. (exemplo: acidentes de trânsito, homicídios, quedas, suicídios). Consequentemente, não há como cotejar os dados de um mesmo caso, mesmo que se trabalhe com esses dois sistemas, embora ambos estejam tratando do mesmo evento. A definição operacional de TCE adotada na pesquisa é outro problema. Trauma no segmento cabeça, incluindo todos os possíveis códigos de lesões da CID - Classificação Internacional de Doenças? $?^{1,2}$ Lesões crânio-encefálicas, portanto, incluindo envoltórios e conteúdo intracraniano? Lesões encefálicas, portanto, restringindo-se a conteúdo intracraniano? Segundo Kraus ${ }^{3}$, nas pesquisas epidemiológicas sobre TCE, os diagnósticos selecionados para inclusão na população de estudo não são uniformes e, consequentemente, as análises comparativas entre elas são difíceis de serem feitas. Isto pode ser constatado em alguns estudos por ele selecionados ${ }^{3}$ e que encontram-se apresentados no Quadro 1.

Além da seleção de determinados diagnósticos, outros critérios são também utilizados. Alguns estudos incluem apenas as vítimas de TCE de gravidade específica, como por exemplo: os somente graves ou somente de trauma leve; só aqueles com TCE, ou todos os politraumatizados com TCE; restrito à TCE devido a uma causa externa específica como o acidente de trânsito de veículo a motor; limitado a crianças com TCE ${ }^{4-9}$. Alguns estudos excluem as vítimas de ferimentos penetrantes, enquanto outros as incluem ${ }^{3,10,11}$. Há também dificuldades para obter índices específicos de morbimortalidade. Alguns estudos não esclarecem, no desenho metodológico, se a base foi populacional. Outros, claramente, descrevem a população ou amostra como sendo de ocorrência, impossibilitando a obtenção de índices com poder de comparação quanto aos riscos. 
Quanto à incidência anual de TCE fatal e não fatal, Kraus³ comenta que não há, nos EUA, estatística precisa. As estimativas publicadas apresentam variações relativamente grandes. $\mathrm{O}$ autor apresenta estimativa em torno de 200/100 000 habitantes. Em Aquitaine (França), em 1986, o índice estimado foi de 281/100 000 habitantes ${ }^{12}$. Em uma determinada região da Austrália, a incidência anual de TCE, foi estimada em aproximadamente 100/100 000 habitantes ${ }^{13}$. No Brasil, Masini ${ }^{14}$ estimou, para Brasília, no ano de 1991, incidência de TCE de 341/100 000 habitantes. Em São Paulo, nenhuma estimativa da incidência anual de TCE, em nível populacional, foi encontrada.

Os problemas de ordem metodológica são menores quando se trata de índices de mortalidade por haver possibilidade de estimativa se os dados estiverem apresentados quanto à causa externa e a natureza da lesão.

Embora vários sejam os problemas de ordem metodológica nos estudos de morbimortalidade por TCE, considera-se importante apresentar as possibilidades de análise com as bases de dados disponíveis no nosso meio, assim como a descrição de algumas características dessas vítimas, no município de São Paulo, onde a mortalidade por acidentes e violências é alta e o TCE é uma das lesões mais frequentes.

O presente estudo tem como objetivos: analisar a incidência de TCE, em vítimas residentes e internadas na rede hospitalar do Município de São Paulo, segundo tipo de lesão, idade, sexo, tempo de permanência hospitalar e condições de saída hospitalar; estimar a mortalidade por TCE, dentre as mortes por causas externas, nesse mesmo local e período.

\section{MÉTODO}

Foram utilizados dados de internações hospitalares e de óbitos de residentes no Município de São Paulo, em 1997.

\section{Internações}

Os dados de morbidade provêm do Sistema de Informações Hospitalares - SIH/SUS. Estima-se que esse sistema seja responsável por $80 \%$ da assistência médico-hospitalar prestada à população brasileira, representando cerca de 12000000 internações/ano, em mais de 6000 unidades hospitalares ${ }^{15}$. No Brasil, as hospitalizações pagas pelo sistema público de saúde são conhecidas por meio de um instrumento, preenchido nos hospitais, obrigatório para a internação dos pacientes e para posterior reembolso. Trata-se da Autorização de Internação Hospitalar (AIH) que contém todas as informações relativas às internações, como dados demográficos, diagnósticos, procedimentos realizados e custos. Esse documento permite o conhecimento do perfil da morbidade atendida por essa parcela do sistema e, de maneira importante, o cálculo dos custos das diferentes causas de internação. Cabe destacar que, para os atendimentos de pronto socorro, não existe o preenchimento de AIH o que, especificamente, quanto às lesões e envenenamentos, subestima a real importância da morbidade por essas causas. Entretanto, a massa de dados, conforme salientado, é de tal ordem que permite uma visão bastante ampla desse panorama.

Para os internados em 1997, a classificação em vigor era a CID-9¹ e os códigos selecionados foram:

800 - fratura da abóbada do crânio

801 - fratura da base do crânio

803 - outras fraturas do crânio e as não especificadas

804 - fraturas múltiplas atingindo o crânio ou face com outros ossos

850 a 854 - traumatismos intracranianos, exceto os associados com fratura de crânio:

850 - concussão do cérebro

851 - laceração e contusão cerebrais

852 - hemorragias subaracnoidiana, subdural e extradural consequentes a traumatismos

853 - outras hemorragias intracranianas e as não especificadas, consequentes a traumatismo

854 - lesão traumática intracraniana de outra natureza e de natureza não especificada

Óbitos

Os dados foram obtidos do PROAIM - Programa de Aprimoramento das Informações de Mortalidade, que funciona junto ao Serviço Funerário do Município de São Paulo, desde 1990, e que, em nível municipal, neste Estado, alimenta o Sistema Oficial de Informações de Mortalidade do Ministério da Saúde (SIM/MS). 
Visto que, desde 1996, está em vigor, para os dados de mortalidade, a 10ª Revisão da CID, OMS-1985², foram selecionadas as mortes decorrentes de causas externas, que tiveram como causa básica as relacionadas no capítulo XX, códigos V01 a Y98. Nesse estudo, eles foram agrupados em "acidentes de transporte", "demais acidentes", "suicídios", "agressões" e um grupo residual para o qual não foi possível conhecer a causa básica da morte, sabendo-se apenas que se tratava de causa externa (aqui denominado "ignorado").

Como o ano de 1997 representa uma fase de transição para a CID, visto que, para a codificação dos dados de mortalidade já estava em vigor a $10^{\mathrm{a}}$ Revisão (desde 1996) e para a morbidade ainda vigia a $9^{\mathrm{a}}$ Revisão (a $10^{\mathrm{a}}$ só entrou em vigor em 1998), foi necessário estabelecer, uma correspondência entre os códigos dos mesmos eventos, em cada uma delas, tanto com relação à natureza da lesão, quanto ao tipo de acidente/violência que originou essa lesão.

\section{População}

A população do Município de São Paulo, em 1997, foi obtida pelo IBGE, sendo que a sua distribuição, segundo sexo e idade, obedeceu às mesmas proporções apresentadas na Contagem de $1996^{16}$.

\section{RESULTADOS E DISCUSSÃO}

No Município de São Paulo, em 1997, tendo como bases residência e ocorrência, nessa localidade, 29717 pacientes foram internados devido a lesões e envenenamentos. Destes, 3635 (12\%) pacientes tinham TCE como diagnóstico principal. A taxa de internação foi 0,36/1 000 habitantes.

Masini ${ }^{14}$, utilizando como critério de inclusão o local de ocorrência do trauma, verificou que, em Brasília, em 1991, num hospital que centraliza atendimentos de vítimas de trauma, foram atendidos 13521 pacientes. Destes, $5436(40 \%)$ apresentavam TCE, e $808(6 \%)$ foram internados. A taxa de internação foi, portanto, 0,5/1 000 habitantes. Entretanto, é preciso salientar que, nesse estudo ${ }^{14}$, foram computadas as vítimas cujo trauma ocorreu em Brasília, incluindo tanto os residentes como os não residentes naquela localidade. Assim, embora, à primeira vista, as taxas pareçam similares, é preciso lembrar que a de São Paulo, incluiu somente pacientes cujo trauma ocorreu nessa cidade e eram residentes na mesma localidade. As características da população estudada, quanto à idade e sexo, estão apresentadas em gráfico1 (Fig 1).

A faixa etária de maior incidência foi a de menores que 10 anos (20,3\%), seguida das de 20 a 29 anos e 30 a 39 anos (16,9\% e 16,1\%, respectivamente). Colli, et al. ${ }^{17}$ também detectaram a incidência

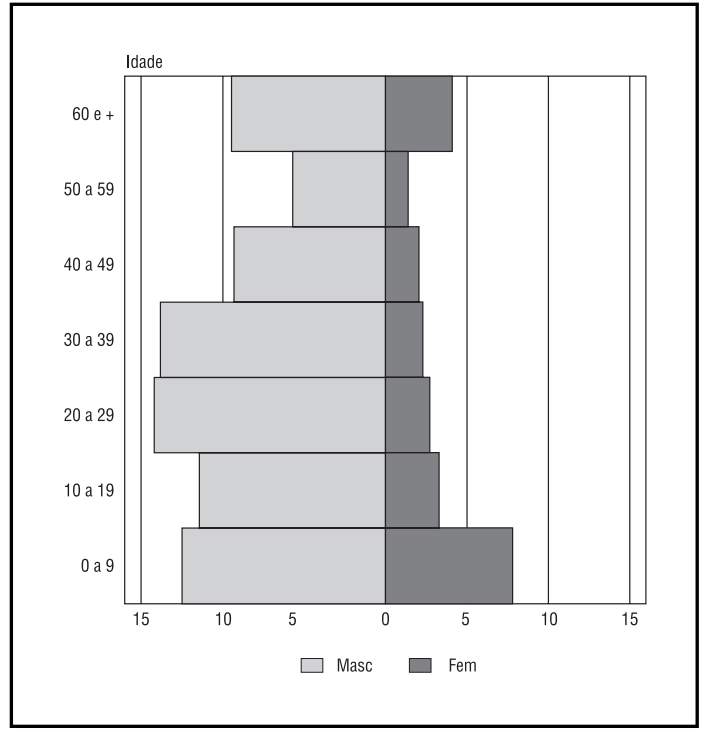

Fig 1. Pacientes internados por TCE segundo sexo e idade, Município de São Paulo, 1997. mais alta na faixa etária de 0 a 10 anos, seguida daquela de 21 a 30 anos.

O predomínio do adulto jovem nas vítimas de TCE é corroborado pela literatura ${ }^{3,14,17}$ e está relacionado com acidentes e violências, atingindo principalmente a faixa populacional de maior atividade laborativa. Por outro lado, principalmente nas publicações da década de 90, observa-se que há uma crescente preocupação com a alta incidência de TCE em menores que 10 anos $^{4-7,9}$.

Quanto ao sexo, houve predomínio do masculino, alcançando 2784(76,6\%) do total de pacientes. Este predomínio do sexo masculino foi maior, principalmente, nas faixas etárias predominantes compreendidas entre 20 e 49 anos. Tais resultados são compatíveis com as observações da literatura ${ }^{1,4,7,10,13,14,17}$. 
Tabela 1. Pacientes internados devido TCE segundo diagnóstico principal. Município de São Paulo, 1997.

\begin{tabular}{lcc}
\hline Diagnóstico principal & $\mathrm{N}$ & $\%$ \\
\hline 800 - fratura da abóbada do crânio & 45 & 1,2 \\
801 -fratura da base do crânio & 32 & 0,9 \\
803 - outras fraturas do crânio & 22 & 0,6 \\
804 - fraturas múltiplas crânio/face & 4 & 0,1 \\
850 - concussão do cérebro & 1038 & 28,6 \\
851 - laceração e contusão cerebral & 192 & 5,3 \\
852,853 - hemorragias & 509 & 14,0 \\
854 - lesão traumática intracraniana de outra natureza & 1793 & 49,3 \\
Total & 3635 & 100 \\
\hline
\end{tabular}

A distribuição por diagnóstico principal é apresentada na Tabela 1.

Quase metade dos internados (49,3\%) tinha o diagnóstico principal classificado no código 854 , que engloba a lesão traumática craniana de outra natureza e os não especificados. O que chama a atenção, além da alta frequência, é a imprecisão desse diagnóstico, ocorrendo o mesmo no diagnóstico que foi o terceiro mais frequente. Os diagnósticos com códigos 852 e 853, ou seja, as hemorragias subaracnóidea, subdural, extradural consequentes a traumatismo e outras hemorragias intracranianas e as não especificadas ficaram nesta posição, somando 14,0\%. Já o segundo diagnóstico mais frequente foi o de código 850, ou concussão cerebral, fato esperado visto que nos TCE é importante a manutenção de um período de observação para detecção de lesões secundárias que demandem internação $(28,6 \%)$. Como já mencionado, a faixa etária de maior frequência foi a de crianças até 10 anos, 737 pacientes. Nessa, os diagnósticos predominantes foram: 850 - concussão cerebral com $304(41,2 \%)$ pacientes e 854 - lesão traumática intracraniana de outra natureza e os não especificados com $335(45,5 \%)$ pacientes. Eles recaíram mais naqueles menores que 5 anos e apresentaram predomínio do sexo masculino.

Embora de certa forma, alguma inferência sobre a gravidade do trauma possa ser feita a partir destes diagnósticos principais , a sua precisão deixa a desejar. A identificação e a pontuação de cada uma das lesões apresentadas pelo paciente e posterior cálculo da gravidade do trauma, utilizando o sistema AIS/ISS (Abbreviated Injury Scale/ Injury Severity Score) e a ECGl (Escala de Coma de Glasgow), conferem maior precisão quanto à gravidade do trauma e tem sido bastante utilizada em $\mathrm{TCE}^{4,6-9,14,17-19}$. Há, portanto necessidade de recuperação de dados do prontuário do paciente, de estudo retrospectivo ou contar com protocolos específicos.

Pela Tabela 2, pode-se verificar que há grande predomínio de internação de 1 a 7 dias (72,5\%). O tempo de internação hospitalar variou entre menos que 1 dia e 374 dias (média 2,2 e DP 0,58; mediana 2,9 e moda 2,0). Novamente, pode-se perceber que tendo somente o diagnóstico principal, a relação com os graus de gravidade do trauma fica prejudicada, exceto naqueles que morreram durante a internação.

A taxa de mortalidade hospitalar dos internados por TCE, no presente estudo, foi $10,2 \%$. A taxa constatada por Colli et al. ${ }^{17}$ foi $5,7 \%$ mas, incluiu todos os atendidos por TCE (internados ou ambulatoriais). O índice de mortalidade hospitalar tem sido usado para avaliar e comparar resultados entre várias instituições quanto à qualidade do cuidado. Resultados de alguns estudos selecionados mostram variação de $4 \%$ a $25 \%$ sendo que, os mais altos índices incluem pacientes assaltados ou atingidos por arma de fogo ${ }^{3}$. No presente estudo, tanto os traumas fechados como os penetrantes encontram-se incluídos. Algumas precauções devem ser tomadas ao usar este índice para estimar a mortalidade hospitalar, devido à prática de internação seletiva diferenciada por hospital; triagem 
Tabela 2. Pacientes internados devido TCE segundo diagnóstico principal e dias de permanência no hospital. Município de São Paulo, 1997.

\begin{tabular}{|c|c|c|c|c|c|c|c|c|c|c|}
\hline \multirow{3}{*}{ Diagnóstico principal } & \multicolumn{10}{|c|}{ Dias de permanência } \\
\hline & \multicolumn{2}{|c|}{$<1$} & \multicolumn{2}{|c|}{$1|-| 7$} & \multicolumn{2}{|c|}{$8|-| 30$} & \multicolumn{2}{|c|}{$>31$} & \multicolumn{2}{|c|}{ Total } \\
\hline & $\mathrm{N}$ & $\%$ & $\mathrm{~N}$ & $\%$ & $\mathrm{~N}$ & $\%$ & $\mathrm{~N}$ & $\%$ & $\mathrm{~N}$ & $\%$ \\
\hline 800-804 - Fraturas & 1 & $(0,1)$ & 74 & $(2,0)$ & 26 & $(0,7)$ & 2 & $(0,1)$ & 103 & $(2,8)$ \\
\hline 850 - Concussão & 59 & $(1,6)$ & 859 & $(23,6)$ & 99 & $(2,8)$ & 21 & $(0,6)$ & 1038 & $(28,6)$ \\
\hline 851 - Laceração/contusão & 15 & $(0,4)$ & 118 & $(3,3)$ & 51 & $(1,4)$ & 8 & $(0,2)$ & 192 & $(5,3)$ \\
\hline 852-3 - Hemorragias & 16 & $(0,4)$ & 290 & $(8,0)$ & 175 & $(4,8)$ & 28 & $(0,8)$ & 509 & $(14,0)$ \\
\hline 854 - Lesão traumática & 97 & $(2,7)$ & 1296 & $(35,7)$ & 325 & $(8,9)$ & 75 & $(2,0)$ & 1793 & $(49,3)$ \\
\hline Total & 188 & $(5,2)$ & 2637 & $(72,5)$ & 676 & $(18,6)$ & 134 & $(3,7)$ & 3635 & (100) \\
\hline
\end{tabular}

Tabela 3. Pacientes internados devido TCE segundo idade e condições de saída hospitalar. Município de São Paulo, 1997.

\begin{tabular}{lcccccc}
\hline Idade & \multicolumn{5}{c}{$\begin{array}{c}\text { Condições de saída } \\
\text { Alta }\end{array}$} & \multicolumn{2}{c}{ Óbito } & \multicolumn{2}{c}{ Total } \\
\hline & $\mathrm{N}$ & $\%$ & $\mathrm{~N}$ & $\%$ & $\mathrm{~N}$ & $\%$ \\
\cline { 2 - 8 } $0|-| 9$ & 727 & $(22,3)$ & 10 & $(2,7)$ & 737 & $(20,3)$ \\
$10|-| 19$ & 502 & $(15,4)$ & 32 & $(8,6)$ & 534 & $(14,7)$ \\
$20|-| 29$ & 536 & $(16,4)$ & 77 & $(20,8)$ & 613 & $(16,9)$ \\
$30|-| 39$ & 525 & $(16,1)$ & 59 & $(15,9)$ & 584 & $(16,1)$ \\
$40|-| 49$ & 351 & $(10,8)$ & 61 & $(16,5)$ & 412 & $(11,3)$ \\
$50|-| 59$ & 220 & $(6,7)$ & 38 & $(10,2)$ & 258 & $(7,1)$ \\
$>60$ & 403 & $(12,3)$ & 94 & $(25,3)$ & 497 & $(13,6)$ \\
Total & 3264 & $(100)$ & 371 & $(100)$ & 3635 & $(100)$ \\
\hline
\end{tabular}

diferenciada para seguimento dos pacientes; variação no período de seguimento para determinação da fatalidade ou alguma combinação desses três itens ${ }^{3}$. Neste estudo, o período medido, foi da internação à data de saída do hospital. Além disso, ao utilizar dados de um sistema como a AIH para estudos de mortalidade hospitalar, como o feito neste estudo, é preciso sempre lembrar que ele está limitado aos pacientes internados, ou seja, não abrange aqueles que foram atendidos no Serviços de Emergência e, a seguir, dispensados. Também não inclui os que morreram no local do evento. Particularmente num dos tipos de causa externa que é o homicídio, a perda é grande, dado que a maior proporção desses óbitos ocorre no próprio local.

Quanto à idade, nos que morreram, constatou-se um predomínio das faixas etárias de adultos jovens, ou seja, daqueles entre 20 e 49 anos, como pode ser constatado pelos dados da Tabela 3 . Na mesma tabela pode-se ainda observar que, embora a faixa etária de 0 a 9 anos tenha sido a de maior proporção de pacientes, ela foi a que apresentou o menor percentual dentre os que morreram. $\mathrm{O}$ inverso ocorreu na faixa etária de maior que 60 anos, se analisada quanto ao percentual de óbitos.

No tocante ao sexo, a predominância do masculino ficou mantida, somando $311(83,8 \%)$ do total de óbitos. Outro fato a ser assinalado refere-se ao momento do óbito durante a hospitalização. 
Tabela 4. Internações por diagnóstico principal (natureza da lesão) e diagnóstico secundário (causa externa) no Município de São Paulo, julho a dezembro de 1998.

\begin{tabular}{|c|c|c|c|c|c|c|c|c|c|c|c|c|c|c|}
\hline \multirow{3}{*}{$\begin{array}{l}\text { Diagnóstico } \\
\text { principal (tipo } \\
\text { de lesão) }\end{array}$} & \multicolumn{12}{|c|}{ Diagnóstico secundário } & \multirow{2}{*}{\multicolumn{2}{|c|}{ Total }} \\
\hline & \multicolumn{2}{|c|}{$\begin{array}{l}\text { Acidente de } \\
\text { transporte }\end{array}$} & \multicolumn{2}{|c|}{ Outros acidentes } & \multicolumn{2}{|c|}{$\begin{array}{c}\text { Tentativas de } \\
\text { suicídio }\end{array}$} & \multicolumn{2}{|c|}{ Agresssões } & \multicolumn{2}{|c|}{$\begin{array}{l}\text { Intenção } \\
\text { ignorada }\end{array}$} & \multicolumn{2}{|c|}{$\begin{array}{l}\text { Complicações } \\
\text { assistência } \\
\text { médica }\end{array}$} & & \\
\hline & $\mathrm{n}^{\circ}$ & $(\%)$ & $\mathrm{n}^{\mathrm{o}}$ & $(\%)$ & $\mathrm{n}^{\mathrm{o}}$ & $(\%)$ & $\mathrm{n}^{\circ}$ & $(\%)$ & $\mathrm{n}^{\circ}$ & $(\%)$ & $\mathrm{n}^{\circ}$ & $(\%)$ & $\mathrm{n}^{\circ}$ & $(\%)$ \\
\hline $\begin{array}{l}\text { Fratura de } \\
\text { crânio e face } \\
(\mathrm{S}-02)^{*}\end{array}$ & 146 & $(24,5)$ & 269 & $(45,1)$ & - & - & 157 & $(26,3)$ & 21 & $(3) 5$, & 3 & $(0,5)$ & 596 & $(100)$ \\
\hline $\begin{array}{l}\text { Traumatismo } \\
\text { intracraniano } \\
(\mathrm{S}-06)^{*}\end{array}$ & 400 & $(29,1)$ & 812 & $(59,1)$ & 3 & $(0,2)$ & 99 & $(7,2)$ & 48 & $(3,5)$ & 12 & $(0,9)$ & 1374 & (100) \\
\hline Ambos & 546 & $(27,7)$ & 1081 & $(54,9)$ & 3 & $(0,2)$ & 256 & $(13,0)$ & 69 & $(3,5)$ & 15 & $(0,8)$ & 1970 & $(100)$ \\
\hline $\begin{array}{l}\text { Todas as } \\
\text { demais lesões e } \\
\text { envenenamentos }\end{array}$ & 3740 & $(21,2)$ & 11327 & $(64,2)$ & 176 & $(1,0)$ & 925 & $(5,2)$ & 393 & $(2,2)$ & 1087 & $(6,2)$ & 17646 & $(100)$ \\
\hline Total & 4286 & $(21,8)$ & 12408 & $(63,3)$ & 179 & $(0,9)$ & 1181 & $(6,0)$ & 462 & $(2,4)$ & 1102 & $(5,6)$ & 19616 & (100) \\
\hline
\end{tabular}

* Organização Mundial da Saúde. CID-10². Fonte: DATASUS (dados brutos não publicados).

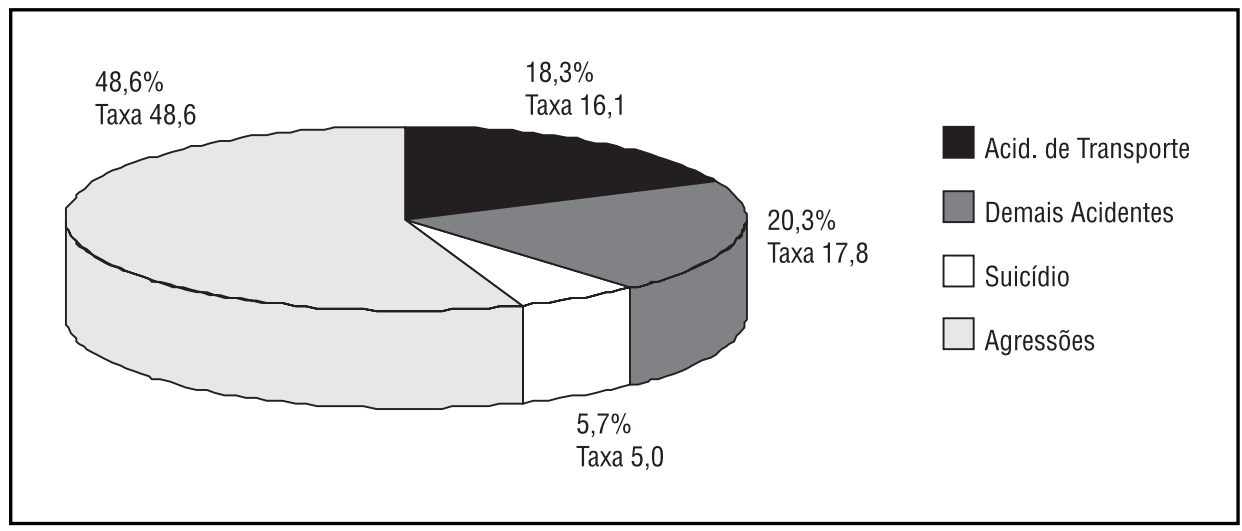

Fig 2. Mortalidade proporcional e coeficiente de mortalidade por causas externas segundo tipo. Município de São Paulo, 1997.

Da totalidade de óbitos, 75 (20,2\%) e 226 (60,9\%) ocorreram até 1 dia e entre 1 e 7 dias de internação, denotando a gravidade do TCE nesses pacientes.

Há, portanto, evidências de que, também no Município de São Paulo, a morbi-mortalidade por TCE é alta. Ainda que a assistência integrada pré-hospitalar e hospitalar de emergência continue sendo aprimorada, bem como as intervenções nas vítimas de TCE contem com recursos cada vez mais avançados, a prevenção, como forma de diminuir estas ocorrências, deve ser melhor administrada. Para isto, é preciso que, além da natureza da lesão, haja indicação clara quanto ao tipo de causa externa que a originou, referência para programas de prevenção. Nesse sentido, a partir de 1998, o SIH/SUS passou a integrar, no seu banco de dados, além da natureza da lesão (diagnóstico principal), a causa externa como diagnóstico secundário. 
No segundo semestre de 1998, foram internados nos hospitais do Município (cobertura do SUS), 19618 pacientes com diagnóstico principal "lesões e envenenamentos"(capítulo XIX da CID-10), o que representou $8,1 \%$ do total das internações. Concomitantemente, todas apresentaram também, registro de diagnóstico secundário, ou seja, o tipo de acidente ou violência que desencadeou o evento (Tabela 4). Pela Tabela 4, é possível verificar algumas características específicas quando a natureza da lesão é relacionada com a causa externa. Do total de 1970 internações por TCE, no Município de São Paulo, computadas por ocorrência (residentes e não residentes), aquelas devidas a "outros acidentes"(em geral, quedas) foram as mais frequentes $(54,9 \%)$. Em segundo lugar figuraram os acidentes de transporte $(27,7 \%)$, havendo, em ambos, predomínio do traumatismo intracraniano em relação à fratura de crânio e face. Já nas agressões (tentativas de homicídio), terceiro em frequência de internação, o diagnóstico principal recaiu na fratura de crânio e face.

É interessante enfatizar que o perfil que se apresenta ao estudar os pacientes internados é diferente daquele revelado pela mortalidade por causas externas (Fig 2). Nesta, o homicídio predomina sobre os demais tipos, representando mais da metade da morte por acidentes e violências $(55,7 \%)$. Os acidentes de transporte correspondem a 19\%. Nos internados, 55\% são devido a quedas, aparecendo, em segundo lugar, os acidentes de transporte com um percentual bastante elevado $(21,8 \%)$. As tentativas de homicídio somam $6 \%$, mostrando que a morte provocada por essa causa ocorre predominantemente no próprio local do evento, não chegando o indivíduo a ser hospitalizado.

O coeficiente de mortalidade por acidentes e violências, para o Município, em 1997, foi de $87,3 / 100000$ habitantes. De acordo com o tipo de causa externa, correspondeu a 48,6 para homicídios, 16,0 para acidentes de transporte e todos os outros tipos somaram 22,7/100 000 habitantes. O sistema oficial de informação sobre mortalidade não permite que, rotineiramente, se possa obter a proporção de TCE na população que morre. Com base em algumas pesquisas específicas ${ }^{20-26}$, foi possível estimar que, das 8333 mortes violentas ocorridas e de residentes no Município, pelo menos 2590 , podendo chegar a 3885 , seriam decorrentes de TCE. Esses números fazem prever uma taxa mínima de mortalidade por TCE, de 26,2 e máxima de 39,3/100 000 habitantes.

Uma estimativa de mortalidade por TCE, nos EUA, baseada em dados publicados, abrangendo a década de 60 até início dos anos 90, principalmente o período de 1980-91, indicou como sendo de aproximadamente 30/100 000/ano ou 52\% de todas as mortes por trauma ${ }^{3}$. Em um outro estudo, igualmente nos EUA, no período de 1979-86, foi estimado que a proporção de TCE, dentre as mortes por trauma, esteja em $28 \%$, com variação de $23 \%$ a $44 \%{ }^{11}$. Pode-se, pois, constatar que, a proporção exata de mortos por TCE é desconhecida.

\section{CONCLUSÕES}

Nas vítimas internadas devido a TCE, o predomínio do sexo masculino foi evidente e a faixa etária mais atingida foi a de até 10 anos, seguida pelas faixas de 20-29 anos e 30-39 anos. A taxa de internação foi $0,36 / 1000$ habitantes, com tempo de internação predominando em até 7 dias $(79,7 \%)$ e a taxa de mortalidade hospitalar foi 10,2\%. Os diagnósticos principais predominaram em lesões do conteúdo intracraniano (códigos 850-854). A mortalidade por TCE, em nível populacional, foi estimada entre 26 e $39 / 100000$ habitantes.

A análise das fontes de dados oficiais disponíveis sobre morbidade hospitalar e mortalidade evidenciam que, para poder melhor delinear o perfil epidemiológico dos TCE, é necessário ter tanto os dados de natureza da lesão como de causa externa nos dois sistemas. É preciso acrescentar, no sistema de dados de mortalidade, cuja base é a causa externa, o diagnóstico de natureza da lesão, a exemplo do que vem sendo feito com os dados de morbidade que trazia só a natureza da lesão e que, a partir de 1998, passou a incluir a causa externa

Para poder aprofundar as análises quanto aos efeitos desse agravo, em nível hospitalar, seria oportuno e importante ter um protocolo único com dados coletados, prospectivamente, em hospitais de referência para trauma, em âmbito regional ou nacional. Elas forneceriam as diretrizes básicas, tanto para os programas de prevenção como para as intervenções específicas da área assistencial. 


\section{REFERÊNCIAS}

1. Organização Mundial da Saúde. Classificação Internacional de Doenças,1975. 9ª Revisão. São Paulo: CBCD 1978:475-659.

2. Organização Mundial da Saúde CID-10. Classificação Estatística Internacional de Doenças e Problemas Relacionados à Saúde. 10ª Revisão. São Paulo, EDUSP, 1998:969-1076.

3. Kraus JF. Epidemiology of head injury. In Cooper PR (ed). Head injury. 3Ed. Baltimore: Williams \& Wilkins,1993:1-25.

4. Barros JL, Pereira LCM, Cavalcante JAF, et al. Avaliação do protocolo de traumatismo craniencefálico na unidade de terapia intensiva do Hospital de Base do Distrito Federal. Arq Bras Neurocirurg 1994;13:64-68.

5. Durkin MS, Olsen S, Barlow B, et al. The epidemiology of urban pediatric neurological trauma: evaluation of, and implication for, injury prevention programs. Neurosurgery 1998;42:300-310.

6. Emanuelson I, Wendt LV. Epidemiology of traumatic brain injury in children and adolescentes in south-western Sweden. Acta Paediatr 1997;6:730-735.

7. Henry PC, Hauber RH, Rice M. Factors associated with closed head injury in a pediatric population. J Neurosci Nurs 1992;24:311-316.

8. Martins EV, Bruggemann M, Coutinho M. Estudo prospectivo de 200 casos de TCE grave na grande Florianópolis. Rev Bras Terap Intens 1997;9:175-180.

9. Semple PL, Bass DH, Peper JC Severe head injury in children: a preventable but forgotten epidemic. SAMJ 1998;88:440-444.

10. Gennarelli TA, Champion HR, Copes WS, et al. Comparison of mortality, morbidity, and severity of 59,713 head injured patients with 114,447 patients with extracranial injuries. J Trauma 1994;37:962-968.

11. Sosin D, Sacks JJ, Smith SM. Head injury: associated deaths in the United States from 1979 to 1986. JAMA 1989;262:251-255.

12. Tiret L, Huasher E, Thicoipe M, et al. The epidemiology of head trauma in Aquitaine (France), 1986: a community-based study of hospital admission and deaths. Int J Epidemiol 1990;19:133-140.

13. Tate RL, McDonald S, Lulham JM. Incidence of hospital-treated traumatic brain injury in an Australian community. Aust NZJ Public Health 1998;22:419-423.

14. Masini M. Perfil epidemiológico do traumatismo crânio-encefálico no Distrito Federal em 1991. J Bras Neurocirug 1994;5:61-68.

15. Levcovitz E, Pereira TRC. SIH/SUS (Sistema AIH): uma análise do sistema público de remuneração de internação hospitalar no Brasil, 1983-1991. Rio de Janeiro: Universidade Estadual do Rio de Janeiro/Instituto de Medicina Social, 1993.

16. IBGE, 1996. Contagem da população, 1996. Município de São Paulo por sexo e idade. S/d [mimeografado]

17. Colli BO, Sato T, Oliveira RS, et al. Características dos pacientes com traumatismo crânio-encefálico atendidos no Hospital das Clínicas da Faculdade de Medicina de Ribeirão Preto. Arq Neuropsiquiatr 1997;55:91-100.

18. Acosta JA, Yang JC, Winchell RJ, et al. Lethal injuries and time to death in a level I Trauma Center. J Am Coll Surg 1998; 186:529-533.

19. Sauaia A, Moore FA, Moore EE, et al. Epidemiology of trauma deaths: a reassessment. J Trauma 1995;38:185-193.

20. Andrade SM. Acidentes de transporte terrestre em Londrina, Paraná; análise das vítimas. Tese de Doutorado, Universidade de São Paulo. São Paulo, 1998

21. Gawryszewski VP. A mortalidade por causas externas no Município de São Paulo, 1991. Dissertação de Mestrado, Universidade de São Paulo. São Paulo, 1995.

22. Ladeira RM. Morbi-mortalidade por acidentes de trânsito em cinco hospitais de Belo Horizonte e Contagem, $1994 / 95$. Dissertação de Mestrado, Universidade Federal de Minas Gerais, Belo Horizonte, 1995.

23. Mello Jorge MHP. Mortalidade por causas violentas no município de São Paulo. Tese de Doutorado, Universidade de São Paulo. São Paulo, 1979.

24. Mello Jorge MHP. Investigação sobre a mortalidade por acidentes e violências na infância. Tese de Livre-Docência, Universidade de São Paulo. São Paulo, 1988.

25. Mesquita MA Filho. Morbidade por acidentes de trânsito em Pouso Alegre, Minas Gerais. Tese de Mestrado, Universidade Federal de São Paulo. São Paulo, 1998.

26. Santo AH. Causas múltiplas de morte: formas de apresentação e métodos análise. Tese de Doutorado, Universidade de São Paulo. São Paulo, 1988. 